\title{
Chronic pain - treatment with spinal cord neurostimulation
}

\author{
Author: Brazilian Medical Association, Brazilian Society of Neurosurgery \\ Participants: Arthur Cukiert'; Alexandre Mac Donald Reis²; Antonio Silvinato de Almeida \\ Ricardo dos Santos Simões²; Renata F. Buzzini'; Wanderley Marques Bernardo ${ }^{1}$
}

Final version: March 21, 2017

1. Brazilian Medical Association 2. Brazilian Society of Neurosurgery http://dx.doi.org/10.1590/1806-9282.64.04.299

The Guidelines Project, an initiative of the Brazilian Medical Association, aims to combine information from the medical field in order to standardize producers to assist the reasoning and decision-making of doctors.

The information provided through this project must be assessed and criticized by the physician responsible for the conduct that will be adopted, depending on the conditions and the clinical status of each patient.

\section{METHODOLOGY FOR EVIDENCE COLLECTION}

This guideline followed the pattern of a systematic review with evidence collection based on the movement of Evidence-Based Medicine, in which clinical experience is integrated with the ability of critical analysis, rationally applying scientific information and improving the quality of medical assistance.

We used the structured version of the question synthesized by the PICO process, in which $\mathbf{P}$ stands for patients with chronic pain that have not responded to the conventional treatment; I for spinal cord neurostimulation intervention; $\mathbf{C}$ for comparison between conventional therapy, reoperation, and spinal cord neurostimulation inactive or absent; and $O$ for clinical outcomes.

Through the elaboration of five relevant clinical questions related to the proposed theme, based on the structured question, we identified the keywords that served as the basis for searching the source databases: Medline-PubMed and Embase Cochrane Library. Next, the summaries of the studies were analyzed and, after applied the eligibility criteria (inclusion and exclusion), 13 papers were selected to answer the clinical questions (Appendix I).

\section{MAIN CLINICAL QUESTION:}

Can patients who have not responded to conventional treatment, pharmacological or not (physical therapy, acupuncture, transcutaneous electrical nerve stimulation, psychotherapy), benefit from spinal cord neurostimulation?

\section{SPECIFIC CLINICAL QUESTIONS}

1. What is the effectiveness of percutaneous Spinal Cord Stimulation (SCS) when compared to the conventional treatment for treating post-laminectomy syndrome?

2. What is the effectiveness of percutaneous SCS combined with the conventional treatment when compared to reoperation combined with the conventional treatment for treating post-laminectomy syndrome?

3. What is the effectiveness of percutaneous SCS for treating type I Complex Regional Pain Syndrome (CRPS-I)?

4. What is the effectiveness of percutaneous SCS for treating refractory angina pectoris?

5 . What is the effectiveness of percutaneous SCS for treating Critical Limb Ischemia (CLI)?

\section{GRADES FOR RECOMMENDATION AND LEVELS OF EVIDENCE}

A: Experimental or observational studies of higher consistency.

B: Experimental or observational studies of lower consistency. 
C: Uncontrolled case/study reports.

D: Opinion deprived of critical evaluation, based on consensus, physiological studies or animal models.

\section{OBJECTIVE}

Identify the best evidence currently available related to the use of spinal cord neurostimulation in patients with chronic pain, assessing its impact on clinical outcomes.

\section{CONFLICT OF INTEREST}

There is no conflict of interest related to this review that can be declared by any of the authors.

\section{INTRODUCTION}

Based on electrophysiological findings and clinical data, in 1965 Melzack e Wall ${ }^{1}$ established the principle of sensory interaction as part of the gate control theory. The theory proposes that the stimulation of structures of the Peripheral Nervous System (PNS) creates impulse patterns that reach the Central Nervous System (CNS), where the information is affected by modulation systems before the perception of pain is evoked. The gelatinous substance of the Posterior Horn of the Spinal Cord (PHSC) acts as a modulation element for the afferent impulses, and the posterior funiculus tracts activate brain structures that, by means of descending fibers, are projected in the spinal cord, interfering with the segmental system activity. Pain and other evoked sensations are the results of the balance between the activities of the primary afferents and the inhibited segmental and suprasegmental pathways. The cells of the gelatinous substance act, thus, as a gate, determining the quality of the stimulus that reaches the cells and originates the suprasegmental projection tracts. The theory suggests the existence of regional inhibition mechanisms that allow the improvement of stimuli identification and offer a physiological basis for correlating the psychological aspects, the attention, and the environmental influences related to pain processing.

The first implantations of spinal electrodes for pain control purposes were carried out in 1967 by means of laminectomy. Initially, the unipolar or bipolar electrodes were implanted in the subarachnoid space. Then, in the subdural compartment and, now, it is preferred to be put in the epidural situation. The current electrodes are quadrupolar and the percutaneous implantation technique is most often employed, since it is safer and simpler.

The electrodes are connected to a battery (like a pacemaker generator) to produce a pain control effect by means of the electrical stimulation of the posterior funiculus of the spinal cord at adequate and established levels, according to the metameres involved and the paresthesia reported by the patient.

The neurophysiological mechanisms for pain relief involve, in addition to the neurophysiological activity, neurochemical elements that explain the lasting effects of analgesics (such as, neurotransmitter modulation, impact on the GABA system, CGRP, etc. $)^{2}$.

Spinal cord neurostimulation is intended to reduce the unpleasant sensory experience caused by pain, as well as its functional and behavioral effects.

\section{PRESENTATION OF RESULTS}

Spinal cord neurostimulation

in neuropathic pain

1. What is the effectiveness of percutaneous Spinal Cord Stimulation (SCS) when compared to the conventional treatment for treating post-laminectomy syndrome?

Patients that underwent Spinal Cord Stimulation (SCS) presented at least $50 \%$ of pain relief in the legs $(\mathrm{NNT}=3)$ when compared to the conventional treatment, using the Visual Analogue Scale, in 6 months. ${ }^{3}(\mathbf{B})$

Patients that underwent SCS did not present any statistically relevant difference in relation to the use of opiates when compared to the conventional treatment (NNT = NS), in 6 months. ${ }^{3}(\mathbf{B})$

Patients that underwent SCS presented an improvement (on average) in functional capacity assessed by the Oswestry disability index, in comparison to conventional therapy $(\mathrm{p}<0.001)$, in 6 months. ${ }^{3}(\mathbf{B})$

Patients that underwent SCS presented statistically relevant $(p<0.05)$ benefits (on average) in quality of life measured by the Short Form-36 (SF-36), in all domains, with the exception of physical performance, in comparison to conventional therapy. ${ }^{3}(\mathbf{B})$

After 6 months, $73 \%$ of the patients in the control group (conventional therapy) migrated to the intervention (SCS) group, and 10\% of the intervention group migrated to control. Due to the risk of bias in 
the short-term analysis, we opted for assessing up to 6 months, excluding another publication of the same study that presented a 24 -month analysis. ${ }^{4}(\mathbf{B})$

2. What is the effectiveness of percutaneous SCS combined with the conventional treatment when compared to reoperation combined with the conventional treatment for treating post-laminectomy syndrome?

Patients that underwent SCS presented 50\% of pain relief (McGill Pain Questionnaire checklist) in three years, in comparison to those of reoperation $(\mathrm{NNT}=3, \mathrm{CI}-95 \%=2$ a 14$) \cdot{ }^{5}(\mathbf{B})$

Patients that underwent SCS presented, in three years, a reduction in opioid use, in comparison those of reoperation $(\mathrm{NNT}=3$, CI-95\% $=2$ a 15$) .{ }^{5}(\mathbf{B})$

There were no differences between SCS and reoperation patients regarding the pain related to daily activities or neurological function. ${ }^{5}(\mathbf{B})$

These results should be evaluated with caution, because $54 \%$ of the reoperation group migrated to the SCS, and $21 \%$ of the SCS for the reoperation after 6 months. There are no data available for analysis at 6 months. The analysis was performed per treatment. ${ }^{5}(\mathbf{B})$

3. What is the effectiveness of percutaneous SCS for treating type I Complex Regional Pain Syndrome (CRPS-I)?

The authors evaluated the effectiveness of the SCS combined with physical therapy, comparing it to the sole use of physical therapy for treating CRPS-I at 6,24 , and 60 months. The outcomes considered were: reduction of pain intensity (VAS-O to $10 \mathrm{~cm}$ ), functional score (function, strength, the amplitude of movement), changes in health-related quality of life (HRQoL). Analysis by Intention to Treat..$^{6-8}(\mathbf{B})$

SCS combined with physical therapy was more successful at reducing pain than physical therapy alone, in average, at 6 months $(-2.4 \mathrm{~cm} / 0.2 \mathrm{~cm}$, $\mathrm{p}<0.001)$ and at 2 years $(-2.1 / 0 \mathrm{~cm}, \mathrm{p}=0.001)$, but did not at 5 years $(-1.7 \mathrm{~cm} /-1.0 \mathrm{~cm}, \mathrm{p}=0.25) \cdot{ }^{6-8}(\mathbf{B})$

There was no difference between SCS combined with physical therapy and physical therapy alone with respect to function, strength, and amplitude of movement of the hands and feet $(p>0,05)$, in the assessments at 6,24 , and 60 months.

There was no difference observed in the assessment of change in health-related quality of life (HRQoL) at 6 months (percent variation in HRQoL:
$6 \%$ in intervention [SCS] and 3\% in control [physical therapy], $p=0.58)$, nor at 2 years ( $7 \%$ in SCS and $12 \%$ in physical therapy, $\mathrm{p}=0.41) \cdot{ }^{6-8}(\mathbf{B})$

Out of the 36 patients selected for SCS, 24 (67\%) were implanted with the device and 12 others (33\%), who did not respond to stimulation (paresthesia covering the entire area of pain, 50\% of VAS reduction over a period of 4 days), were analyzed by ITT. The sample size calculation, considering a $90 \%$ ability to detect a $2.3 \mathrm{~cm}$ difference in VAS between treatments with a significance level of $5 \%$, indicated that 34 patients would be needed in the SCS group, as described in the study. ${ }^{6-8}(\mathbf{B})$

\section{Spinal cord neurostimulation in ischemic pain}

\section{What is the effectiveness of percutaneous SCS} for treating refractory angina pectoris?

Patients with refractory angina, high-risk surgical patients, without a good prognosis for myocardial revascularization surgery and with contraindication for percutaneous angioplasty (anatomical alterations and others) were included. Thus, when analyzing SCS versus Myocardial Revascularization Surgery (MRS), at 6 months, there was no difference in reduction, on average, of the number of weekly angina attacks between SCS and MRS ( $p=$ NS). ${ }^{9,10}(\mathbf{B})$

There was no difference in the consumption of short-acting nitrate (dose/week), on average, between SCS and MRS ( $p=$ NS). The myocardial revascularization surgery reduced, on average, the consumption of long-acting nitrate $(\mathrm{p}<0.0001) \cdot{ }^{9,10}(\mathbf{B})$

The myocardial revascularization surgery increases the ability to exercise $(p=0.02)$.

There was no difference in the health-related quality of life between SCS and MRS (Error bars: \pm 1 SEM) - 'Nottingham Health Profile' (NHP) e 'Quality of Life Questionnaire - Angina Pectoris' (QLQAP) $)^{9,10}(\mathbf{B})$

When analyzing SCS versus Myocardial Revascularization Surgery (MRS), at five years, there was no difference in the quality of life between SCS and MRS (Error bars: \pm 1 SEM) ${ }^{9,10}(\mathbf{B})$

There was no difference in survival (Kaplan-Meier) between SCS and MRS. ${ }^{9,10}(\mathbf{B})$

In one study, patients with refractory angina, with no conditions of undergoing percutaneous angioplasty or myocardial revascularization surgery were included and analyzed at 6 weeks. ${ }^{11}(\mathbf{B})$

The was no pain reduction, on average, assessed by VAS $(0-10 \mathrm{~cm})\left[1.1 \mathrm{~cm} \times 0.2 \mathrm{~cm} ; \mathrm{p}=0.29 .{ }^{.1}(\mathbf{B})\right.$ 
In relation frequency of angina attacks (per day), it was reduced, on average, $2.3 \times 3.2(p=0.01) .{ }^{11}(\mathbf{B})$

There was a reduction in nitrate consumption $(-48 \% \times 27 \%, p=0.03)$ and an improvement, on average, of the ability to exercise ( $533 \times 427$, duration in seconds $)(p=0.03) \cdot{ }^{11}(\mathbf{B})$

There was no difference, on average, in the Quality of Life (Linear Analogue Self-Assessment [LASA] scale) $(p=0,10) \cdot{ }^{11}(\mathbf{B})$

The sample of the study is small (13 for SCS and 12 for control [inactive SCS]). Considerable Type II Error. ${ }^{11}(\mathbf{B})$

The authors included in the study patients with refractory angina to whom the myocardial revascularization surgery was considered inadequate or with no good prognosis, assessed at 8 weeks. ${ }^{12}(\mathbf{B})$

The frequency of angina attacks (per week) was reduced, on average, $9.0 \times 13.6(\mathrm{p}<0.05)$ in up to 8 weeks. ${ }^{12}(\mathbf{B})$ The nitrate consumption (weekly average) was reduced, $1.6 \times 8.5(p<0.05)$ in up to 8 weeks..$^{12}(\mathbf{B})$

There was an improvement, on average, in the ability to exercise (cardiac stress test) [duration of the exercise in seconds: $827 \times 694, p<0.002$ ] in up to 8 weeks. ${ }^{12}(\mathbf{B})$ And the quality of life (HRQoL - daily activity score, including social activity) was improved $(\mathrm{p}<0.05)$ in up to 8 weeks. ${ }^{12}(\mathbf{B})$

The small sample size of this study (SCS $=8$ and no SCS=9) entails a relevant Type II Error. The follow-up time is very short (8 weeks). ${ }^{12}(\mathbf{B})$

Patients with refractory angina and reversible myocardial ischemia, however inadequate for conventional revascularization, were analyzed at 3 and 12 months. In relation to the ability to exercise (duration in minutes), there was no improvement, on average, at 3 and 12 months ( $>0.05$ for both measurements). There was no difference, on average, in the quality of life $(p>0.05) \cdot{ }^{13}(\mathbf{B})$

5. What is the effectiveness of percutaneous SCS for treating Critical Limb Ischemia (CLI)?

Diabetic patients with atherosclerotic chronic critical ischemia of the lower limbs, lasting over two weeks, with pain at rest and/or ulceration, for which there was an impossibility or failure of revascularization due to poor circulation (analysis at 18 months). ${ }^{14}(\mathbf{B})$

\section{- Pain (VAS)}

It was not possible to assess pain levels at 18 months due to the loss of $80 \%$ of the control group for this outcome.

\section{- Amputation}

SCS did not reduce the number of amputations up to 18 months (NNT = NS).

- Limb salvage (revascularization of the ischemic limb)

Limb salvage was not improved (NNT $=$ NS) up to 18 months.

Patients with atherosclerotic peripheral arterial occlusive disease in advanced stage (Fontaine IV [ulcer or gangrene]); not all diabetics; with the impossibility of revascularization and severe pain at rest despite analgesic medication (analysis at 12 months). ${ }^{15}(\mathbf{B})$

SCS combined with the use of prostaglandin E1 (intravenously), compared to the exclusive use of prostaglandin E1, did not reduce the number of amputations (smaller and larger) up to 12 months (NNT $=\mathrm{NS}) \cdot{ }^{15}(\mathbf{B})$

Patients with atherosclerotic chronic critical ischemia of the lower limbs, with pain at rest and/or ulceration, for which there was the impossibility of revascularization (analysis at 2 years). ${ }^{16.17}(\mathbf{B})$

There was no difference in the pain assessment (VAS, McGill Pain Questionnaire, pain-rating index) ( $p>0.05)$. Mortality was not reduced, neither was the number of amputations up to 2 years (NNT=NS). There was no difference in limb salvage up to 2 years (Kaplan-Maier; HR = 1.09, CI95\%: 0,59 - 2,03) or in quality of life (Nottingham Health Profile [NHP] and EuroQol) $(\mathrm{p}>0.05) .{ }^{16,17}(\mathbf{B})$

\section{FINAL CONSIDERATIONS}

Spinal cord stimulation should only be considered as a treatment option after the assessment by a multidisciplinary team with experience in chronic pain assessment.

\section{Spinal cord neurostimulation for treating pain with neuropathic origins}

Patients who did not present at least 50\% of VAS improvement in pain levels and did not respond to conventional treatment (pharmacological or not [physical therapy, psychotherapy, acupuncture...]) for a period of at least 6 months.

\section{Post-laminectomy syndrome}

There is evidence of estimated benefits in using SCS in comparison to reoperation, by means 
of pain reduction (at least 50\% in VAS), [NNT=3]; improvement in functional capacity and in the health-related quality of life $(p<0.05)$ in up to 6 months (Level 2B).

\subsection{Complex Regional Pain Syndrome type I}

The use of SCS combined with physical therapy compared to the exclusive use of physical therapy as treatment reduces the pain according to the VAS (Visual Analogue Scale) score, [p $<0,001]$. However, it does not improve the functional assessment (function, strength and amplitude of movement) and the health-related quality of life ( $p>0.05)$ up to 2 years.

\section{Spinal cord neurostimulation for treating} ischemic pain

\subsection{Refractory angina}

In patients with refractory angina for whom the myocardial revascularization surgery was considered inadequate, the SCS, when compared to inactive SCS or no SCS, in up to 8 weeks, reduced the number of angina attacks and improved the ability to exercise. The assessment of the quality of life is inconclusive, since one study shows improvement and the other does not. Due to the small sample size, the type II error is considerable in both studies.

In patients with refractory angina and reversible myocardial ischemia, however inadequate for conventional revascularization, the SCS compared to laser percutaneous myocardial revascularization does not improve the ability to exercise $(p>0.05)$ and there is no difference in quality of life ( $p>0.05)$ in up to 12 months.

In patients with refractory angina; high-risk surgical patients; with no good prognosis for myocardial revascularization surgery and contraindication for percutaneous angioplasty (anatomical alterations and others), the SCS, compared to the myocardial revascularization surgery, in up to 6 months, did not reduce angina attacks and the consumption of nitrates; it did not improve the ability to exercise or the quality of life ( $\mathrm{p}=\mathrm{NS}$ for all outcomes - average results).

In the 5-year analysis, there was no improvement in the quality of life ( $p=N S$ ) and in survival (Kaplan-Meier).

\subsection{Chronic Critical Limb Ischemia}

In diabetic patients with atherosclerotic chronic critical ischemia of the lower limbs lasting for over two weeks, with pain at rest and/or ulceration, for which there was impossibility or failure of revascularization due to poor circulation, the SCS combined with oral analgesic compared to the exclusive use of oral analgesic did not reduce the number of amputations nor did it improve limb salvage $(\mathrm{NNT}=\mathrm{NS})$ in up to 18 months.

In patients with atherosclerotic peripheral arterial occlusive disease in advanced stage (Fontaine IV [ulcer or gangrene]), not all diabetics, with impossibility of revascularization and severe pain at rest despite the use of analgesic medication, the SCS combined with prostaglandin E1, compared to the exclusive use of intravenous prostaglandin, did not reduce the number of amputations (smaller and larger) in up to 12 months.

In patients with atherosclerotic chronic critical ischemia of the lower limbs, with pain at rest and/or ulceration, for which there was impossibility of revascularization, the SCS combined with medical care, when compared to only medical care (analgesics, antithrombotic drugs, vasoactive drugs, local treatment of the ulcer and antibiotics, when necessary) did not reduce the pain ( $p>0.05)$; did not reduce the mortality or the number of amputations (NNT=NS); did not improved the limb salvage (HR = 1.09, CI95\%: 0.59 $2.03)$ and did not improve the quality of life ( $>0.05)$ up to 24 months.

\section{RECOMMENDATION \\ Use of spinal cord neurostimulation in neuropathic pain}

For patients who did not present at least 50\% of improvement in pain levels in the VAS score (Visual Analogue Scale) and who did not respond to conventional treatment (pharmacological or not [physical therapy, psychotherapy, acupuncture...]), for a minimum period of 6 months, there is evidence to support the use of spinal cord neurostimulation for post-laminectomy syndrome and complex regional pain syndrome type I.

\section{Use of spinal cord neurostimulation in ischemic pain}

There is no evidence to support the use of spinal cord neurostimulation in patients with chronic ischemic pain (refractory angina and chronic critical ischemia of the lower limbs with no possibility of revascularization). 


\section{REFERENCES}

1. Melzack R, Wall PD: Pain mechanisms: a new theory. Science 150: 971979, 1965 Merry F, Smith WM, Anderson DJ, Emmens DJ, Choong CK: Cost-effectiveness of spinal cord stimulation in patients with intractable angina. N Z Med / 114: 179-181, 2001

2. Linderoth B, Gazelius B, Franck |, Brodin E: Dorsal column stimulation induces release of serotonin and substance $P$ in the cat dorsal horn. Neurosurgery 31: 289-297, 1992

3. Kumar K, Taylor RS, Jacques L, Eldabe S, Meglio M, Molet J, Thomson S,O'Callaghan |, Eisenberg E, Milbouw G, Buchser E, Fortini G, Richardson J, North RB. Spinal cord stimulation versus conventional medical management for neuropathic pain: a multicentre randomised controlled trial in patients with failed back surgery syndrome. Pain. 2007 Nov;132(1-2):17988. Epub 2007 Sep 12.

4. Kumar K, Taylor RS, Jacques L, Eldabe S, Meglio M, Molet J, Thomson S, O'Callaghan J, Eisenberg E, Milbouw G, Buchser E, Fortini G, Richardson I, North RB. The effects of spinal cord stimulation in neuropathic pain are sustained: a 24-month follow-up of the prospective randomized controlled multicenter trial of the effectiveness of spinal cord stimulation. Neurosurgery. 2008 Oct;63(4):762-70; discussion 770.

5. North RB, Kidd DH, Farrokhi F, Piantadosi SA. Spinal cord stimulation versus repeated lumbosacral spine surgery for chronic pain: a randomized, controlled trial. Neurosurgery. 2005;56(1):98-106; discussion 106-7.

6. Kemler MA, Barendse GA, van Kleef M, de Vet HC, Rijks CP, Furnée $C A$, van den Wildenberg FA. Spinal cord stimulation in patients with chronic reflex sympathetic dystrophy. N Engl J Med. 2000 Aug 31;343(9):618-24

7. Kemler MA, De Vet HC, Barendse GA, Van Den Wildenberg FA, Van Kleef $M$. The effect of spinal cord stimulation in patients with chronic reflex sympathetic dystrophy: two years' follow-up of the randomized controlled trial. Ann Neurol. 2004 Jan;55(1):13-8.

8. Kemler MA, de Vet HC, Barendse GA, van den Wildenberg FA, van Kleef $M$. Effect of spinal cord stimulation for chronic complex regional pain syndrome Type I: five-year final follow-up of patients in a randomized controlled trial. J Neurosurg. 2008 Feb;108(2):292-8.

9. Mannheimer C, Eliasson T, Augustinsson LE, Blomstrand C, Emanuelsson $\mathrm{H}$, Larsson S, Norrsell H, Hjalmarsson A. Electrical stimulation versus coronary artery bypass surgery in severe angina pectoris: the ESBY study. Circulation. 1998 Mar 31;97(12):1157-63.
10. Ekre $O$, Eliasson $T$, Norrsell $H$, Währborg $P$, Mannheimer $C$. Electrical Stimulation versus Coronary Artery Bypass Surgery in Severe Angina Pectoris. Long-term effects of spinal cord stimulation and coronary artery bypass grafting on quality of life and survival in the ESBY study. Eur Heart J. 2002 Dec;23(24):1938-45.

11. Hautvast RW, Dejongste MJ, Staal MJ, van Gilst WH, Lie KI. Spinal cord stimulation in chronic intractable angina pectoris: a randomized, controlled efficacy study. Am Heart ). 1998 Dec;136(6):1114-20.

12. de Jongste MI, Hautvast RW, Hillege HL, Lie KI. Efficacy of spinal cord stimulation as adjuvant therapy for intractable angina pectoris: a prospective, randomized clinical study. Working Group on Neurocardiology. J Am Coll Cardiol. 1994 Jun;23(7):1592-7.

13. McNab D, Khan SN, Sharples LD, Ryan JY, Freeman C, Caine N, Tait S, Hardy I, Schofield PM. An open label, single-centre, randomized trial of spinal cord stimulation vs. percutaneous myocardial laser revascularization in patients with refractory angina pectoris: the SPiRiT trial. Eur Heart J. 2006 May;27(9):1048-53. Epub 2006 Mar 22.

14. |ivegård LE, Augustinsson LE, Holm I, Risberg B, Ortenwall P. Effects of spinal cord stimulation (SCS) in patients with inoperable severe lower limb ischaemia: a prospective randomised controlled study. Eur | Vasc Endovasc Surg. 1995 May;9(4):421-5.

15. Claeys LG, Horsch S. Transcutaneous oxygen pressure as predictive parameter for ulcer healing in endstage vascular patients treated with spinal cord stimulation. Int Angiol. 1996 Dec;15(4):344-9.

16. Klomp HM, Spincemaille GH, Steyerberg EW, Habbema JD, van Urk H. Spinal-cord stimulation in critical limb ischaemia: a randomised trial. ESES Study Group. Lancet. 1999 Mar 27;353(9158):1040-4.

17. Spincemaille GH, Klomp HM, Steyerberg EW, Habbema JD. Pain and quality of life in patients with critical limb ischaemia: results of a randomized controlled multicentre study on the effect of spinal cord stimulation. ESES study group. Eur J Pain. 2000;4(2):173-84.

18. Jadad AR, Moore RA, Carroll D, Jenkinson C, Reynolds D|, Gavaghan D|, et al. Assessing the quality of reports of randomized clinical trials: is blinding necessary? Control Clin Trials 1996; 17:1-12.

19. Kemler MA, Reulen JP, Barendse GA, van Kleef $M$, de Vet $H C$, van den Wildenberg FA. Impact of spinal cord stimulation on sensory characteristics in complex regional pain syndrome type l: a randomized trial. Anesthesiology. 2001 Jul;95(1):72-80. 
APPENDIX I

\section{Structured question}

\begin{tabular}{|l|}
\hline $\begin{array}{l}\text { P - Patients with chronic pain who did not respond to convention- } \\
\text { al treatment }\end{array}$ \\
\hline I - Spinal cord neurostimulation \\
\hline $\begin{array}{l}\text { C - Conventional therapy, reoperation, spinal cord neurostimula- } \\
\text { tion inactive or absent }\end{array}$ \\
\hline O- Clinical outcomes
\end{tabular}

\section{Methodology for evidence search PubMed-Medline}

Strategy 1: ((((Electric Stimulation Therapy) AND Spinal Cord) AND Pain))

Strategy 2: with clinical queries filter (Therapy/Narrow [filter]) AND ((((Electric Stimulation Therapy) AND Spinal Cord) AND Pain))

\section{COCHRANE}

spinal cord AND electric stimulation

\section{Manual search}

No papers were selected from manual search.

\begin{tabular}{l|l} 
DATABASE & NUMBER OF PAPERS \\
\hline Primary \\
\hline PubMed-Medline & $1,347 / 67$ (with Cl. Queries filter) \\
\hline Embase & 249 \\
\hline Secondary & 2 (System Rev.)
\end{tabular}

TABLE 1 - No. of papers returned from the search methodology used in each of the scientific databases

\section{Selection of papers}

The studies were initially selected by title, then by their summary and, lastly, by their complete text, being the last one subject to critical evaluation and the extraction of all results relating to outcomes.

All studies recovered from the primary and secondary databases were evaluated.

From the primary databases, after the initial critical evaluation, were selected: PubMed-Medline (13), Embase (zero), and Cochrane/Lilacs/BVS (zero).

The papers considered for complete reading were critically evaluated following the inclusion and exclusion criteria, based on study performance, PICO, language and availability of the text in its entirety.

Table 2 presents the number of papers assessed by the clinical situation.

\begin{tabular}{|ll|}
\hline CLINICAL INDICATION & NUMBER OF PAPERS \\
\hline Post-laminectomy syndrome & 3 \\
Complex regional pain syndrome type I & 3 \\
Chronic critical limb ischemia & 3 \\
Refractory angina & 4 \\
TOTAL & $\mathbf{1 3}$ \\
\hline
\end{tabular}

TABLE 2 - No. of papers selected for critical evaluation according to clinical indication

\section{Language}

Only studies available in Portuguese, English, or Spanish were included.

\section{According to publication}

Only studies with texts available in its entirety were considered for critical evaluation. Out of the 13 papers considered of critical assessment, none was excluded for not being complete.

\section{Inclusion and exclusion criteria}

The studies were included according to the PICO components, the language, availability of full text and study design.

The designs included varied according to the clinical question, with the selection of the higher level of evidence for each question.

Studies conducted between 1993 and 2011 were included in the assessment.

\section{Critical evaluation and level of evidence}

Only studies with texts available in its entirety were considered for critical evaluation. When, after the inclusion and exclusion criteria were applied, the evidence selected was classified as Randomized Controlled Trial (RCT), it was subjected to a suitable critical evaluation check-list Table 3).

\begin{tabular}{|c|c|}
\hline \multicolumn{2}{|c|}{ Guide for Critical Evaluation of Randomized Controlled Trials (check-list) } \\
\hline $\begin{array}{l}\text { Study data } \\
\text { Reference, study design, Jadad, level } \\
\text { of evidence }\end{array}$ & $\begin{array}{l}\text { Sample size calculation } \\
\text { Estimated differences, power, signifi- } \\
\text { cance level, the total number of patients }\end{array}$ \\
\hline $\begin{array}{l}\text { Patient selection } \\
\text { Inclusion and exclusion criteria }\end{array}$ & $\begin{array}{l}\text { Patients } \\
\text { Recruited, randomized, prognostic } \\
\text { differences }\end{array}$ \\
\hline $\begin{array}{l}\text { Randomization } \\
\text { Description and blinded allocation }\end{array}$ & $\begin{array}{l}\text { Patient follow-up } \\
\text { Time, losses, migration }\end{array}$ \\
\hline $\begin{array}{l}\text { Treatment protocol } \\
\text { Intervention, control, and blinding }\end{array}$ & $\begin{array}{l}\text { Analysis } \\
\text { Intention to treat, analyzed interven- } \\
\text { tion and control }\end{array}$ \\
\hline $\begin{array}{l}\text { Outcomes considered } \\
\text { Primary, secondary, measurement } \\
\text { instrument for the outcome of } \\
\text { interest }\end{array}$ & $\begin{array}{l}\text { Results } \\
\text { Benefits or harmful effects in absolute } \\
\text { data, benefits or harmful effects on } \\
\text { average }\end{array}$ \\
\hline
\end{tabular}

TABLE 3 - Guide for Critical Evaluation of Epidemiological Studies (check-list) 
The critical evaluation of RCT allows to classify it according to the Jadad score ${ }^{18}$, considering Jadad trials $<3$ (three) as inconsistent, and those with score $\geq 3$ (three) consistent.

\section{Extraction of results}

The results regarding the different clinical situations considered will be exposed individually, by means of the following items: clinical question, number of selected works (according to the criteria of inclusion), main reasons for exclusion and synthesis of the evidence available.

References related to studies included and excluded will be arranged in the item References.

For results with available evidence, the population, intervention, outcomes, presence or absence of benefits and/or harmful effects, and controversy will be explicitly defined whenever possible.

In the absence of consistent randomized clinical trials, the synthesis will be limited to the impossibility of sustaining the indication.

Cost-related issues will not be included in the results.

The outcomes considered will be limited to the effectiveness and safety of interventions. The results will be presented, preferably, in absolute data, absolute risk, reduction of absolute risk, and number needed to treat (NNT).

\section{Description of evidence}

Based on well-established indications for spinal cord neurostimulation that indicate a better thera- peutic result, this guideline has assessed its use in the treatment of neuropathic pain (Post-laminectomy syndrome [PLS], Complex Regional Pain Syndrome Type I [CRPS-I]) and of ischemic pain (Critical Limb Ischemia [CLI], Refractory Angina [RA]). ${ }^{19}$

The critical reading of each of the studies was conducted, seeking to identify possible biases that could compromise the internal validity of the study. In the absence of serious invalidating biases, the studies were included in the review and its eventual biases and limitations described individually (Note at the end of each evaluation).

Important biases found during critical analysis of the studies included in this Guideline for the Use of Spinal Cord Neurostimulation correspond to:

1. Small sample size value in most of the studies, leading to a considerable type II error.

2. Risk of bias in the long-term analysis due to migration, which ranged from $54 \%$ to $73 \%$ at 6 months.

Only studies that assessed at least one clinical or clinically relevant outcome (for example, pain, functional capacity, quality of life, amputation, limb salvage) were included. When there was more than one publication of the same study, only the one with clinical outcomes considered relevant was assessed.

There was no subgroup analysis, which increases the possibility of random associations.

As with any neuromodulation technique, the therapeutic results of spinal cord neurostimulation may vary over time as the technology is modified. 